\title{
Cellular Muscle Actuators with Variable Resonant Frequencies
}

\author{
Thomas W. Secord and H. Harry Asada \\ d'Arbeloff Laboratory for Information Systems and Technology \\ Department of Mechanical Engineering \\ Massachusetts Institute of Technology \\ Cambridge, MA 02139, USA \\ Email: $\{$ secord, asada $\} @$ mit.edu
}

\begin{abstract}
This paper presents the design and analysis of a novel variable stiffness and variable resonance actuator based on a cellular arrangement of piezoelectric devices. The cellular muscle actuator design concept is presented followed by a general dynamic model for establishing the theoretical bounds on achievable resonant frequencies. A model that is specific to the proposed design is then formulated to include the effects of parasitic dynamics. The resonance characteristics of a three cell prototype system are identified experimentally. The theoretical model and experimental results agree over a large frequency range and illustrate the variable resonance concept.
\end{abstract}

\section{INTRODUCTION}

Variable stiffness actuators have several unique properties and have been an active research area in robotics during the last two decades. For mobile robots, tunable joint compliance effectively absorbs impulsive reaction forces from the ground and allows a robot to better negotiate with rough terrain. Compliance is also essential in manipulating an object and physically interacting with environments. The compliance in physical contact tasks must often be altered to meet task requirements. Although feedback control is an easy way of varying "servo" stiffness, the bandwidth of a control loop is usually much lower than the desired speed of the physical interaction. Therefore, variable mechanical compliance must be inherent to the actuator's physical construction to meet bandwidth requirements.

Several groups have developed variable stiffness actuators for robotic applications. However, many of these designs are variations on traditional electromechanical motors [1]. Furthermore, much work in this field focuses mainly on legged locomotion (e.g. [2],[3]) and human-robot interaction (e.g. [4]).

In addition to variable stiffness, resonance has also been a key property in robotic systems and can be traced to the first hopping robots of Raibert [5]. The early work of Pratt also demonstrated that traditionally stiff actuators can resonate with a load using series elastic stiffness in the drive train [6]. Resonance has also seen recent use in flapping flight robots [7],[8]. With respect to research focusing on actuators, the work in [9] and [10] examines resonance amplitudes for piezoelectric materials and electrostrictive materials, respectively. Resonance extends into the biomechanics realm as well. Animals, especially humans, prefer gait frequencies that closely correspond to the natural frequencies of their limb pendular motion because it is energetically favorable and it appears to simplify the neural control of movement [11].

The goal of the present work is to combine the beneficial characteristics of variable stiffness and resonance into a single actuator. This paper describes the design of an actuator based on cellular architecture where both variable stiffness and variable resonance properties are independently tunable by changing both stiffness and mass distribution within the actuator. Actuator performance can be improved in many ways if the stiffness and mass properties can be modulated depending on task requirements. Specifically, for a class of periodic motions including flapping, swimming, walking, and running, power efficiency and amplitude of motion can be improved significantly if the frequency of the periodic motion matches the resonance frequency of the combined load and actuator. Tuning resonant frequencies to minimize energy expenditure is especially important for mobile robot applications. The resonance tuning capability is also crucial for energy harvesting applications [12].

The design concept of an actuator with tunable stiffness and mass distribution will be described for cellular muscle actuators consisting of many discrete actuator units. The concept of cellular actuators has been described in previous work of the authors' group. For example, the application of cellular architecture is discussed for shape memory alloy (SMA) systems in [13] and for piezoelectric materials (specifically PZT) in [14]. In this work, existing design strategies for PZT are extended to incorporate variable stiffness.

The paper will first review the cellular architecture concept for PZT and introduce the variable stiffness design. Next, the variable stiffness concept is extended to include variable resonant frequencies and theoretical bounds on the resonant frequencies are established using a simplified model. In the final sections, the model is extended and compared to experimental data. Both the model and experiments show the versatility of the actuator design strategy.

\section{Cellular Architecture and Variable Stiffness DESIGN}

This section summarizes basic principles of variable stiffness cellular actuators (VSCA). A particular PZT cellular 
actuator design will be described to clarify the concepts, yet the basic principles described in this section are applicable to a class of cellular actuators using various smart materials.

\section{A. PZT Cellular Actuators with Large Strain Amplification}

Fig. 1 shows the design concept for a nested-flexure PZT cellular actuator. Each cellular unit consists of a PZT stack and double-layer nested flexures that amplify displacement by a combined factor of 20 (Fig. 1 (a)). This large amplification gain is necessary because PZT has very small inherent strain $(0.1 \%)$. Fig. 1 (b) shows a prototype of serially connected PZT cellular units. The six cellular units yield a $65 \mathrm{~mm}$ net body length and can produce a $7 \mathrm{~mm}$ total free displacement and a $5 \mathrm{~N}$ blocking force.

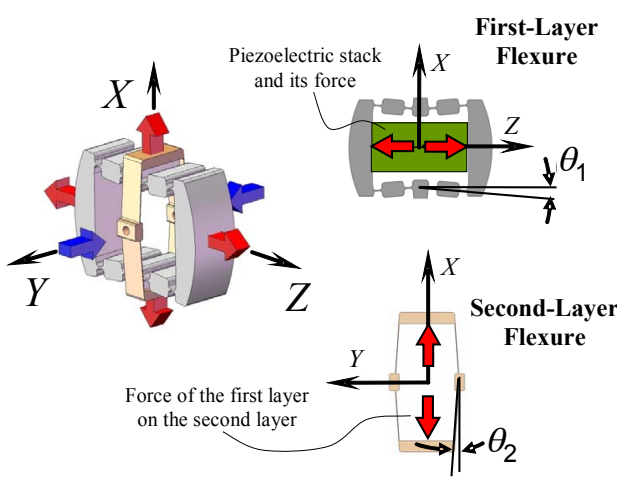

(a)

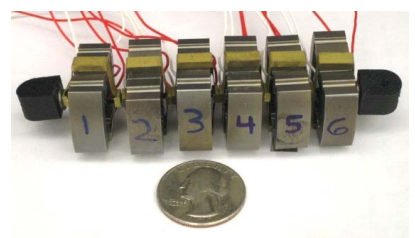

(b)

Fig. 1. (a) Motion of nested amplification flexures. (b) PZT cellular actuator having 6 serially connected cells.

From Fig. 1 (a), observe that as a voltage is applied to the PZT stacks, the first layer flexure is pushed outward along the $Z$ direction, which results in an outward amplified displacement in the $X$ direction provided that $\theta_{1} \ll 1$. The second layer flexure is also pushed outward in the $X$ direction, which results in a further amplified displacement in the inward $Y$ direction with a small $\theta_{2}$. Therefore, a contraction force is generated along the $Y$-axis output as the PZT stacks are activated. This contractile double-layer flexure design provides a muscle-like motion and allows the connection of multiple units in series without buckling of the flexures.

\section{B. Variable Stiffness Mechanism}

Fig. 2 shows a modification to the above design to achieve variable stiffness. Outside the second layer flexure is a rigid structure that limits the stroke of the output displacement in the $Y$ direction. When the PZT is not activated, the output node of the second layer flexure rests on the stroke limiting beam. The output node movement is also limited when an excessive tensile force acts on the output node. As the applied PZT voltage increases, the output node is pulled inward and is detached from the stroke limiting beam.

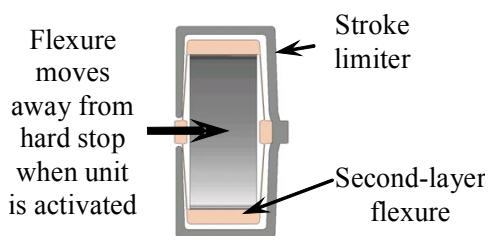

Fig. 2. Design of a variable stiffness PZT-based cell. The system consists of two strain amplification layers. The second layer flexure incorporates a stroke limiting beam.

Fig. 3 is a schematic diagram depicting the mechanism of the cellular unit with a stroke limiter. The outer thin line indicates the stroke limiter, while the vertical thick line shows the output node of the flexure that rests on the stroke limiter when the PZT is inactive (Fig 3 (a)). As the PZT force $f_{p}$ increases, the thick vertical line detaches from the limiter (Fig 3 (b)). In the ON state, the equivalent compliance seen at the output nodes is $1 / k$.

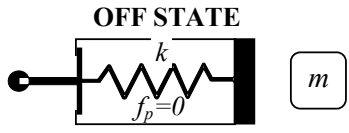

(a)

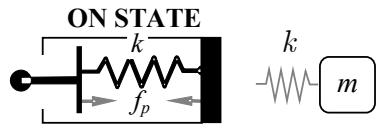

(b)
Fig. 3. Schematic model of a variable stiffness, PZT-actuated cell. (a) OFFstrain limited rigid state. (b) $\mathrm{ON}$ - compliant state.

The compliance characteristics associated with the cellular unit are shown in Fig. 4. The compliance of a single cell is variable in the sense that it can be either zero or a finite value $c=1 / k$. To demonstrate the approach, it is assumed that the compliance follows the ideal curve denoted by the solid line in Fig. 4. In reality, the hard stop imposed by the flexure still affords some finite compliance $c_{f}$. Furthermore, the compliance will actually increase as the cell becomes contracted due to the geometric nonlinearity of the flexure; this is shown as the dashed line in the figure. The nonlinearity is assumed to be negligible within the operation range $0<y<y_{l}$ as shown in the figure.

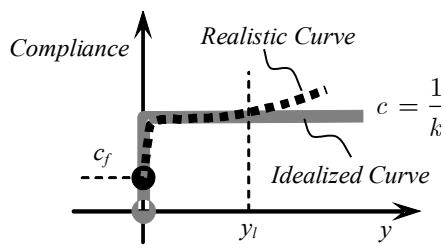

Fig. 4. Compliance characteristics of a variable stiffness PZT-actuated cell.

\section{Serial, Parallel, and Antagonistic Connections}

The above cellular units can be connected in series, parallel, or antagonistic configurations, creating diverse stiffness characteristics as a collective sum. The most fundamental VSCA 
is a serial connection of $N$ units, as shown in Fig. 1 (b). This arrangement is referred to as a strand. For simplicity, assume that the stiffness of an OFF state unit is infinitely large, while stiffness in the ON state is a constant $k$. If $n$ units are $\mathrm{ON}$ and the others are OFF, then the resultant stiffness of an $N$-unit strand reduces to

$$
k_{S}=\frac{k}{n} \quad 1 \leq n \leq N
$$

Next, consider a parallel arrangement of strands. As shown in Fig. 5 (a), $N_{p}$ strands of $N$ serially connected units may be arranged in parallel. If the $i^{t h}$ strand of serially connected units has stiffness $k_{S, i}$, the resultant stiffness of the entire system is given by

$$
k_{P}=\sum_{i=1}^{N_{p}} k_{S, i} .
$$

To accommodate the stiffness to a desired value, one can determine the number of parallel strands, $N_{p}$, and the number of ON units $n_{i}$ in each strand of serial units.

The $N_{p}$ strands can be divided into two sets of strands to form an agonist-antagonist arrangement. As shown in Fig. 5 (b), both the agonist and antagonist strands contribute to an output stiffness as a direct sum: $k_{\text {out }}=k_{P, a g}+k_{P, a n}$. The antagonistic arrangement allows for varying stiffness independent of position, as in the case of natural skeletal muscles [15]. However, unlike skeletal muscles, the stiffness seen at the output of a VSCA will decrease as more cells are activated.

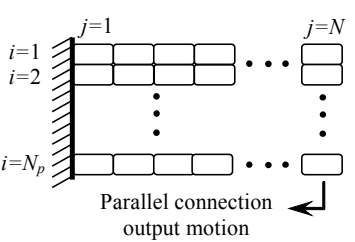

(a)

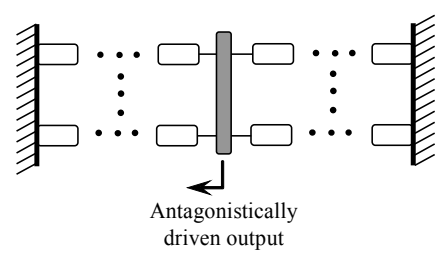

(b)
Fig. 5. (a) Parallel arrangement of cellular units. Note that one end of the actuator is shown connected to ground only for clarity, but myriad other boundary conditions are possible. (b) Antagonistic pairing of actuator strands.

Any VSCA implementation must possess two fundamental characteristics: 1) each cellular unit has discrete stiffness states and can be selectively switched ON or OFF and 2) the units are connected to form an aggregate output. Within this framework many implementations are possible. The next section presents the general dynamic properties of VSCAs and shows how these properties lead to variable resonant frequencies.

\section{VARiable Resonant Frequencies}

\section{A. Principle}

The resonant frequencies of a collection of cellular units can be varied by exploiting the cellular architecture. The key behavior is that turning on a specific number of units to accommodate the static stiffness and displacement still allows for a multitude of ON-OFF unit combinations, each of which exhibit different vibration modes with distinct resonant frequencies. Although the total number of $\mathrm{ON}$ state units remains the same, the resonant frequencies may be different depending upon which units are in the ON state.

To illustrate the basic concept, recall the simplified dynamic model shown in Fig. 3. Here, the distributed mass of the entire single-unit structure is lumped together and modeled as a single mass, $m$, while the compliance is lumped into a single compliance element with spring constant $k$. Consider three of these units connected serially as shown in Fig. 6. Suppose that two out of the three units are turned on so that the static stiffness of the serial connection is $k_{S}=k / 2$. There are three different ways of selecting two units to turn on and they are shown in the upper portion of Fig. 6. Depending upon which units are on, the assembly dynamic behavior is different.

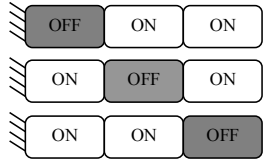

$$
\begin{aligned}
& \text { DYNAMICS } \\
& \exists m \text { in } m^{k}{ }^{k} m \\
& \text { STATICS } \\
& \exists w^{k} m m w^{k} m \\
& \exists M \cdot M \cdot k^{S}=\frac{k}{2} \\
& \exists M^{k} m M^{k} m \\
& \exists M \cdot M \cdot k^{S}=\frac{k}{2} \\
& \exists M \cdot M \cdot k^{S}=\frac{k}{2}
\end{aligned}
$$

Fig. 6. Diverse ON-OFF state distributions of three serially connected units attached to ground.

Since the system in Fig. 6 has two degrees of freedom, there are two distinct vibration modes and resonant frequencies. Table I shows the resonant frequencies for each of the three ON-OFF states with $n=2$. The first configuration, OFF-ON$\mathrm{ON}$, provides the highest resonant frequency overall, while the lowest occurs for the third configuration, ON-ON-OFF. Furthermore, for the first configuration, the grounded mass does not participate dynamically at all, whereas in the second and third cases the OFF unit participates as a translating mass in different parts of the strand.

Since all three cases have the same static stiffness $k_{S}$ but different resonance properties, this example demonstrates that stiffness and resonant frequencies can be changed independently. Although the resonant frequencies do not vary continuously, multiple choices are available for different task requirements. As the number of cellular units increases, the number of possible stiffness levels and resonant frequencies increases. The next subsection describes a simple model for the general case of $N$ serially connected units.

\section{B. Basic Model of Serial Connection Dynamics}

Consider a single serially connected strand of $N$ cellular units. Suppose that $n$ units are in ON state so that $n$ springs are detached from the stroke limiters. This creates an $n$ degree-of-freedom system with each d.o.f. corresponding to a single lumped mass that moves independently. This means 


\begin{tabular}{ccc}
\hline \hline Configuration & $\frac{\omega_{1}}{\sqrt{k / m}}$ & $\frac{\omega_{2}}{\sqrt{k / m}}$ \\
\hline 1) OFF-ON-ON & 0.62 & 1.62 \\
2) ON-OFF-ON & 0.54 & 1.31 \\
3) ON-ON-OFF & 0.47 & 1.51 \\
\hline \hline
\end{tabular}

TABLE I

FIRST AND SECOND NATURAL FREQUENCIES OF EACH OF THE THREE CONFIGURATIONS

that $N-n$ OFF-state units are rigidly connected to other units as illustrated in Fig 7. Let $x_{i}$ be the position of the $i^{\text {th }}$ combined masses and $\mathbf{x}$ be a vector collectively representing the $n$ lumped mass displacements: $\mathbf{x}=\left[x_{1}, x_{2}, \ldots, x_{n}\right]^{T}$.

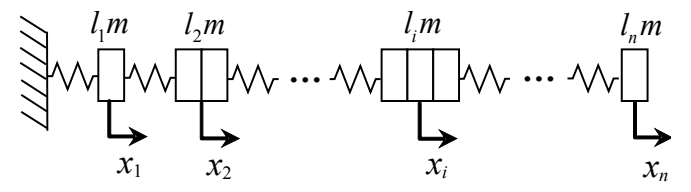

Fig. 7. Simplified dynamic model.

The $i^{\text {th }}$ lumped mass consists of $l_{i}$ cellular units with total mass of $l_{i} m$ with $l_{i} \in \mathbb{N}$. From Fig. 7, the equations of motion are obtained as

$$
m_{i} \ddot{x}_{i}=-2 k x_{i}+k x_{i-1}+k x_{i+1}, \quad 2 \leq i \leq n-1,
$$

and for $i=1$ and $i=n$,

$$
m_{1} \ddot{x}_{1}=-2 k x_{1}+k x_{2}, \quad m_{n} \ddot{x}_{n}=-k x_{n}+k x_{n-1} .
$$

These equations can be arranged into vector-matrix form:

$$
\mathbf{M} \ddot{\mathbf{x}}+\mathbf{K x}=\mathbf{0}
$$

where $\mathbf{M}=m \cdot \operatorname{diag}\left(l_{1}, l_{2}, \ldots, l_{n}\right)=m \mathbf{L}$ and

$$
\begin{aligned}
\mathbf{K} & =k\left(\begin{array}{cccc}
2 & -1 & & 0 \\
-1 & 2 & \ddots & \\
& \ddots & 2 & -1 \\
0 & & -1 & 1
\end{array}\right) \\
& =k \cdot \mathbf{S} .
\end{aligned}
$$

\section{Dynamic Range of Serially-Connected Cells}

It is now of interest to examine how widely the resonant frequencies can be varied for a given static stiffness. The maximum and minimum resonant frequencies of serially connected cellular units are obtained using the model from the previous subsection. A more complete dynamic model for a specific implementation will be obtained in IV to include the effects of distributed mass and damping.

The squared resonant frequencies of the $n$ d.o.f. mass-spring system are given by the eigenvalues of matrix product $\mathbf{M}^{-1} \mathbf{K}$ :

$$
\omega_{j}^{2}=\lambda_{j}\left(\mathbf{M}^{-1} \mathbf{K}\right)=\frac{k}{m} \lambda_{j}\left(\mathbf{L}^{-1} \mathbf{S}\right) .
$$

The $n$ eigenvalues of the matrix are ordered from the minimum to the maximum and written as

$$
\lambda_{\min }\left(\mathbf{M}^{-1} \mathbf{K}\right) \leq \lambda_{2}\left(\mathbf{M}^{-1} \mathbf{K}\right) \leq \cdots \leq \lambda_{\max }\left(\mathbf{M}^{-1} \mathbf{K}\right)
$$

Note that for a fixed number of ON-state units, $n$, the stiffness matrix, $\mathbf{K}$, remains the same regardless of the arrangement of ON-OFF units. The mass matrix, $\mathbf{M}$, on the other hand, varies depending on the ON-OFF arrangement within the strand. Therefore, the above $\lambda_{\min }\left(\lambda_{\max }\right)$ can be further decreased (increased) by changing the ON-OFF unit configurations and thereby changing the mass matrix.

Given a specific static stiffness $k_{S}$, which will uniquely determine $n$, consider the set of all mass matrices associated with the possible distributions of $N$ cells into $n$ clusters of masses:

$$
\begin{gathered}
\mathfrak{M}_{\mathfrak{n}}=\left\{\mathbf{M}=m \cdot \operatorname{diag}\left(l_{1}, \ldots, l_{n}\right) \mid \sum_{i=1}^{n} l_{i}=N-p\right. \\
\left.p=0, \ldots, N-n ; l_{i} \geq 1\right\}
\end{gathered}
$$

The number of possible arrangements of $n$ ON units within a strand is equivalent to the cardinality of $\mathfrak{M}_{n}$ and will be denoted by $P_{n}$ where $P_{n}=\left(\begin{array}{l}N \\ n\end{array}\right)$.

Notice that the subset of $\mathfrak{M}_{n}$ defined by $p=0$ in (9) is the set of all possible mass matrices assuming that the first unit in the strand is in the ON state. For these cases, all of the cell masses will participate dynamically. The subset of $\mathfrak{M}_{n}$ defined by $p=1$ corresponds to the unit connected to ground in the OFF state. In this case, the mass of the first unit in the chain does not participate dynamically. This pattern continues for $p>1$ until $p=N-n$.

Let $\sigma_{\min }(n)$ be the minimum of the positive square root of $\lambda_{\min }\left(\mathbf{M}^{-1} \mathbf{K}\right)$ with respect to all $\mathbf{M} \in \mathfrak{M}_{n}$ :

$$
\sigma_{\min }^{2}(n)=\min _{\mathbf{M} \in \mathfrak{M}_{n}} \lambda_{\min }\left(\mathbf{M}^{-1} \mathbf{K}\right)
$$

$\sigma_{\min }(n)$ provides the lowest resonant frequency among all the ON-OFF unit distributions. Similarly, let

$$
\sigma_{\max }^{2}(n)=\max _{\mathbf{M} \in \mathfrak{M}_{n}} \lambda_{\max }\left(\mathbf{M}^{-1} \mathbf{K}\right)
$$

$\sigma_{\max }(n)$ gives the highest possible resonant frequency.

Recall that minimum and maximum eigenvalues of $\mathbf{M}^{-1} \mathbf{K}$ are given, respectively, by the minimum and maximum of the Rayleigh quotient:

$$
\lambda_{\min }\left(\mathbf{M}^{-1} \mathbf{K}\right)=\min _{\mathbf{x}} \frac{\mathbf{x}^{T} \mathbf{K} \mathbf{x}}{\mathbf{x}^{T} \mathbf{M} \mathbf{x}},
$$

and

$$
\lambda_{\max }\left(\mathbf{M}^{-1} \mathbf{K}\right)=\max _{\mathbf{x}} \frac{\mathbf{x}^{T} \mathbf{K} \mathbf{x}}{\mathbf{x}^{T} \mathbf{M} \mathbf{x}},
$$

where min and max are taken assuming $\mathbf{x} \in \mathbb{R}^{n}$. 
Using (12) and (13), one can prove the following proposition concerning the highest resonant frequency $\sigma_{\max }$ and the ON-OFF unit distribution that provides $\sigma_{\max }$.

\section{Proposition 1:}

Let $\mathbf{M}=m \mathbf{L}$ and $\mathbf{K}=k \mathbf{S}$ be, respectively, the $n \times n$ mass and stiffness matrices of $N$ serially-connected cellular units among which $n$ units are active (turned ON). The highest resonant frequency occurs when the first $N-n$ units are turned OFF. This maximum frequency is given by

$$
\sigma_{\max }=\sqrt{\frac{k}{m} \lambda_{\max }(\mathbf{S})}
$$

Proof:

Since $\mathbf{M} \geq m \mathbf{I}, \forall \mathbf{M} \in \mathfrak{M}_{n}$,

$$
\begin{aligned}
\lambda_{\max }\left(\mathbf{M}^{-1} \mathbf{K}\right) & =\max _{\mathbf{x}} \frac{\mathbf{x}^{T} \mathbf{K} \mathbf{x}}{\mathbf{x}^{T} \mathbf{M} \mathbf{x}} \\
& \leq \max _{\mathbf{x}} \frac{k \mathbf{x}^{T} \mathbf{S} \mathbf{x}}{m \mathbf{x}^{T} \mathbf{I} \mathbf{x}} \\
& =\frac{k}{m} \lambda_{\max }(\mathbf{S}) .
\end{aligned}
$$

Therefore,

$$
\underset{\mathbf{M} \in \mathfrak{M}_{n}}{\arg \max } \lambda_{\max }\left(\mathbf{M}^{-1} \mathbf{K}\right)=m \mathbf{I} .
$$

Hence, the arrangement where the first $N-n$ units are turned OFF yields $\sigma_{\max }$.

For the lowest possible resonant frequency, $\sigma_{\min }$, the following proposition applies.

\section{Proposition 2:}

The arrangement having all the inactive units placed at the unconstrained end of the serial connection gives the lowest un-damped resonant frequency $\sigma_{\min }$.

Proof:

The $n$ d.o.f. undamped mass-spring system with fixedfree boundary conditions and a mass distribution $l_{1} m, l_{2} m, \ldots, l_{n} m$ has a fundamental vibration mode shape $\Phi_{1}$ with monotonically increasing mass displacement amplitudes $\bar{x}_{i}$ from the fixed end to the unconstrained end $(i=1,2, \ldots, n)$. Without loss of generality, the mode shape has been taken as positive. This fact can be shown using the necessary conditions on the fix-free mode shape as described in [16]. Thus, the displacement amplitudes in the first mode shape satisfy

$$
0 \leq \bar{x}_{1} \leq \bar{x}_{2} \leq \ldots \leq \bar{x}_{n} .
$$

The lowest resonant frequency $\sigma_{\min }$ is the frequency of the fundamental vibration mode that has a monotonically increasing modal vector from the fixed end to the unconstrained end, according to (17). Therefore, $\sigma_{\min }$ is given by minimizing the Rayleigh quotient for all $0 \leq x_{1} \leq x_{2} \leq \cdots \leq x_{n}$,

$$
\sigma_{\min }^{2}(n)=\min _{\substack{\mathbf{M} \in \mathfrak{M}_{n} \\ \mathbf{x} \in \mathbb{R}^{n}}} \frac{k \mathbf{x}^{T} \mathbf{S} \mathbf{x}}{\mathbf{x}^{T} \mathbf{M} \mathbf{x}}=\min _{\substack{\mathbf{M} \in \mathfrak{M}_{n} \\ 0 \leq x_{1} \leq \cdots \leq x_{n}}} \frac{k \mathbf{x}^{T} \mathbf{S x}}{\mathbf{x}^{T} \mathbf{M} \mathbf{x}}
$$

Without loss of generality, one can assume $x_{i} \geq 0 i=$ $1,2, \ldots, n$ in minimizing the Rayleigh quotient. Among all the mass distributions, the one having the most masses at the unconstrained end maximizes the denominator of the quotient for all $x_{i}$ satisfying the monotonically increasing modal vector condition in (17). Therefore,

$$
\min _{\substack{M \in \mathfrak{M}_{n} \\ 0 \leq x_{1} \leq \cdots \leq x_{n}}} \frac{k \mathbf{x}^{T} \mathbf{S} \mathbf{x}}{\mathbf{x}^{T} \mathbf{M x}}=\min _{0 \leq x_{1} \leq \cdots \leq x_{n}} \frac{k \mathbf{x}^{T} \mathbf{S} \mathbf{x}}{\mathbf{x}^{T} \mathbf{M}_{0} \mathbf{x}},
$$

where $\mathbf{M}_{0} \triangleq m \cdot \operatorname{diag}(1,1, \ldots, N-n+1)$. This represents the case where all of the inactive units are placed at the unconstrained end.

\section{Detailed Dynamic Model for PZT-Based VSCA}

The analysis in the previous section provides useful insights as to the mechanism of variable resonant frequencies and their variable range. This section will consider a specific implementation of the VSCA and extend the previous analysis to account for distributed mass, damping, and compliance of the stroke limiter for the PZT cell design. The model of this section can be used for any set of meaningful boundary conditions and can be applied to arbitrary parallel or antagonistic connections.

\section{A. Single Cell Dynamic Model}

A detailed lumped parameter model for the PZT-based design is shown in Fig. 8. This model includes the mass $m$ of the hard stop beam as well as its stiffness $K$. The lower case symbol for the hard stop mass indicates that $m<M$. Likewise, the symbol $K$ for the stroke limiting beam stiffness indicates that $K$ is much larger than a single flexure's stiffness $k$. The stiffness $k$ does vary slightly with displacement, but this variation is assumed to be small for the purpose of model development. The viscous damping elements are taken as constant with a value $b$. The damping elements represent losses in the flexures and are interposed between the output nodes of the cell. The damping of the flexures as well as any damping in the load will shift the resonant frequencies away from their undamped counterparts. However, this shift is small insofar as $b$ is relatively small. The PZT-generated force at the output of the second layer is again denoted by $f_{p}$. Each cell in an actuator is subject to two tensile forces $T_{m}$ and $T_{M}$. The tension $T_{M}$ is the tension acting at the output node. Note that it will be useful to express the output node tension as the sum of a preload component $T_{M, P L}$ and a time varying component $\tilde{T}_{M}: T_{M}=T_{M, P L}+\tilde{T}_{M}$.

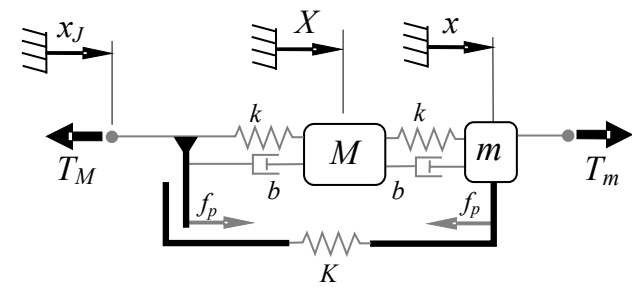

Fig. 8. Single cell model that includes mass distribution, parasitic damping $b$, and parasitic stiffness $K$ of the stroke limiting beam. 
Each cell requires three generalized coordinates to describe its configuration with respect to an inertial reference frame. The first coordinate, $x$, determines the position of the hard stop mass. The second coordinate, $X$, determines the position of the second layer unit suspended mass. The third coordinate, $x_{J}$, determines the location of the output node. Note that if a second unit is connected to the output node, then $x_{J}$ is equal to the inertial displacement of the second unit's hard stop mass.

\section{B. Piezoelectric Induced Force}

The generation of the self-equilibrating force $f_{p}$ is achieved through PZT stack expansion and application of the customdesigned nested flexures. For simplicity of analysis, the assembly of the piezoelectric stack and flexures is treated as a two port element, having one port in the electrical domain and the other port in the mechanical domain at the point of application in the lumped model of Fig. 8. The constitutive law for the generation of the force may be written in a structural stiffness form as

$$
\left\{\begin{array}{c}
V_{p z t} \\
-f_{p}
\end{array}\right\}=\left(\begin{array}{cc}
\kappa_{11} & -\kappa_{12} \\
-\kappa_{12} & \kappa_{22}
\end{array}\right)\left\{\begin{array}{c}
Q_{p z t} \\
\Delta X
\end{array}\right\},
$$

where $V_{p z t}$ is the voltage applied to the piezoelectric stacks, $Q_{p z t}$ is the charge accumulated on the stacks (neglecting hysteresis between applied voltage and charge), $\Delta X=x-x_{J}$, and the elements of the matrix $\kappa_{11}, \kappa_{12}$, and $\kappa_{22}$ represent generalized stiffness constants. The minus sign preceding $f_{p}$ accounts for the model sign convention. Each of the stiffness constants has a physical meaning that is important in the design of the actuator cell. However, for brevity, the $\kappa_{i j}$ constants will not be discussed explicitly. Solving for $f_{p}$ from (20) yields

$$
f_{p}=\left(\frac{\kappa_{12}}{\kappa_{11}}\right) V_{p z t}-\left(\frac{\kappa_{11} \kappa_{22}-\kappa_{12}^{2}}{\kappa_{11}}\right) \Delta X .
$$

\section{Cell Recruitment}

The condition for turning a cell ON may be stated in terms of the applied voltage as

$$
V_{\min }<V_{p z t}<V_{\max },
$$

where $V_{\max }$ is the maximum voltage as specified by the material manufacturer and $V_{\min }$ is the minimum voltage required to move the detachable flexure beams away from the hard stop (Fig 2). Based on the model in IV-B and a force analysis of the output node, the value of $V_{\min }$ is found to be

$$
V_{\text {min }}=\frac{\kappa_{11}}{\kappa_{12}}\left[T_{M, P L}+\tilde{T}_{M}+\beta \Delta X\right],
$$

where $\beta=\left(\frac{\kappa_{11} \kappa_{22}-\kappa_{12}^{2}}{\kappa_{11}}-k\right)$. From (23), observe that the minimum ON voltage is reduced when $\kappa_{11}$ is reduced, $\kappa_{12}$ is increased, or $T_{M, P L}$ is decreased. Eq. (23) also shows that the dynamically generated component of the force acting between actuators $\tilde{T}_{M}$ makes the minimum ON voltage time varying. Therefore, it is crucial that operating conditions are selected for the actuator so that inadvertent switching from ON to OFF or OFF to ON is avoided because this would result in structural chattering and unwanted internal vibrations.

\section{Assembled System Equations of Motion}

The equations of motion for an assembled system may now be obtained by isolating three cells within a rectangular array of dimensions $N_{p} \times N$ as previously discussed in II-C. The equations of motion are formulated for the $i j^{t h}$ cell, with $i$ as the strand index and $j$ as the index of a cell within a strand.

Three separate cases need to be considered because the forces acting on the mass $m$ of the $i j^{\text {th }}$ cell and the $i j+1^{t h}$ cell will be different depending upon the states of these two adjacent cells. For the ON-ON case, the equations of motion are

$$
\left\{\begin{array}{c}
f_{p, i j-1}-f_{p, i j}+k\left(X_{i j-1}-x_{i j}\right)+b\left(\dot{X}_{i j-1}-\dot{x}_{i j}\right) \\
-k\left(x_{i j}-X_{i j}\right)-b\left(\dot{x}_{i j}-\dot{X}_{i j}\right)=m \ddot{x}_{i j} \\
k\left(x_{i j}-X_{i j}\right)+b\left(\dot{x}_{i j}-\dot{X}_{i j}\right) \\
-k\left(X_{i j}-x_{i j+1}\right)-b\left(\dot{X}_{i j}-\dot{x}_{i j+1}\right)=M \ddot{X}_{i j} .
\end{array}\right.
$$

For the OFF-ON case, the equations become

$$
\left\{\begin{array}{c}
f_{p, i j-1}-f_{p, i j}+k\left(X_{i j-1}-x_{i j}\right)+b\left(\dot{X}_{i j-1}-\dot{x}_{i j}\right) \\
+K\left(x_{i j-1}-x_{i j}\right)-k\left(x_{i j}-X_{i j}\right) \\
\quad-b\left(\dot{x}_{i j}-\dot{X}_{i j}\right)=m \ddot{x}_{i j} \\
k\left(x_{i j}-X_{i j}\right)+b\left(\dot{x}_{i j}-\dot{X}_{i j}\right) \\
-k\left(X_{i j}-x_{i j+1}\right)-b\left(\dot{X}_{i j}-\dot{x}_{i j+1}\right)=M \ddot{X}_{i j} .
\end{array}\right.
$$

And for the OFF-OFF case, the equations are

$$
\left\{\begin{array}{c}
f_{p, i j-1}-f_{p, i j}+k\left(X_{i j-1}-x_{i j}\right)+b\left(\dot{X}_{i j-1}-\dot{x}_{i j}\right) \\
+K\left(x_{i j-1}-x_{i j}\right)-k\left(x_{i j}-X_{i j}\right) \\
-b\left(\dot{x}_{i j}-\dot{X}_{i j}\right)-K\left(x_{i j}-x_{i j+1}\right)=m \ddot{x}_{i j} \\
k\left(x_{i j}-X_{i j}\right)+b\left(\dot{x}_{i j}-\dot{X}_{i j}\right) \\
-k\left(X_{i j}-x_{i j+1}\right)-b\left(\dot{X}_{i j}-\dot{x}_{i j+1}\right)=M \ddot{X}_{i j} .
\end{array}\right.
$$

For any set of linear boundary conditions, the system equations of motion may be written in the standard vibratory form as

$$
\mathbf{M} \ddot{\mathbf{q}}+\mathbf{B} \dot{\mathbf{q}}+\mathbf{K}(t) \mathbf{q}=\mathbf{Q}(t),
$$

where $\mathbf{q}$ is the generalized coordinate vector. For simplicity, the second term in (21) is assumed to be small and therefore

$$
f_{p, i j} \approx\left(\frac{\kappa_{12}}{\kappa_{11}}\right) V_{p z t, i j}
$$

Thus, the vector $\mathbf{Q}$ is the vector containing each cell's voltage inputs scaled by the ratio of the stiffness constants $\kappa_{12}$ and $\kappa_{11}$. Both the input $\mathbf{Q}(t)$ and the stiffness matrix $\mathbf{K}(t)$ depend on the ON-OFF arrangement within each strand, thereby making the dynamics equations a switched linear system.

Rearrangement of (24) to (26) leads to a row-wise construction of the mass, stiffness, and damping matrices in (27). Notice that the equations of motion for the masses $M$ 
do not change even when a cell is OFF. Furthermore, the equations corresponding to the large masses $M$ are unforced. Hence, a convenient block diagonal form of the stiffness and damping matrices may be constructed. The model structure can be readily extended to include any load behaving as a linear mechanical impedance and this extension is considered explicitly in the next section.

\section{EXPERIMENTAL RESULTS}

\section{A. Equipment and Procedure}

The cellular PZT design described in II-B was used to construct a three unit VSCA. The identical units were connected in series to give a net length of $41 \mathrm{~mm}$. The actuator was then connected to a plastic cantilever, which provided the tension necessary for operation and acted as a linear spring-massdamper load.

The complete experimental apparatus is shown in Fig. 9. As shown in the figure, vertical deflections of the cantilever, denoted by $x_{L}$, were measured using a MicroEpsilon laser sensor with a resolution of $1 \mu \mathrm{m}$. Voltages were applied to the piezoelectric stacks using a Cedrat CA-45 amplifier.

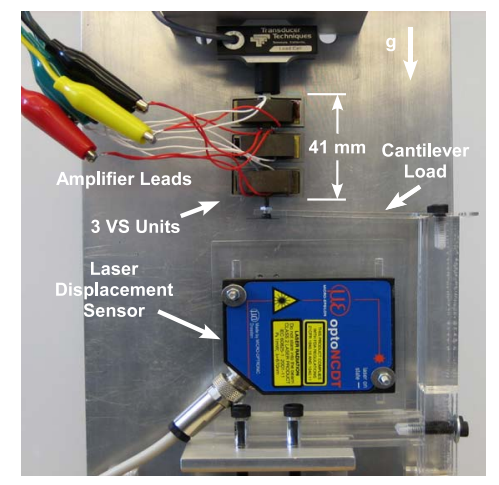

Fig. 9. Experimental apparatus for measuring dynamic properties of a 3-cell system.

A chirp voltage input was used to obtain the frequency response characteristics of the actuator. The chirp signal ranged from $5 \mathrm{~Hz}$ to $150 \mathrm{~Hz}$ and contained a DC offset of $100 \mathrm{~V}$ to assure that the conditions in (22) were satisfied. The maximum voltage used was $150 \mathrm{~V}$. The experimental results for system frequency response were obtained using the ratio of windowed power spectra from the input voltage and output displacement. All measured signals were sampled at $1 \mathrm{kHz}$ with a National Instruments data acquisition board.

Two representative test cases were selected to illustrate the tunability of resonant frequencies. For the first case, the top unit (the uppermost unit in Fig. 9) was turned ON and the lower two units were kept OFF. For the second case, the middle unit was turned $\mathrm{ON}$ while the top and lower unit were kept OFF. Units were maintained in the OFF state by disconnecting their leads from the amplifier. The fundamental resonant frequency is compared for both cases in the next subsection.

\section{B. Experimental and Theoretical Model Comparison}

Before considering the experimental results, the model from IV-D was used to generate a theoretical response for comparison with the data. The cantilever beam shown in Fig. 9 was modeled as a mass, $m_{L}$, connected to ground through a parallel arrangement of a spring and dashpot having values of $k_{L}$ and $b_{L}$, respectively. The resulting model structure for the entire system is given by the following matrix equation:

$$
\begin{aligned}
\mathbf{M} \ddot{\mathbf{q}}+b\left(\begin{array}{cc}
2 \mathbf{I}_{3 \times 3} & \mathbf{A} \\
\mathbf{A}^{T} & 2 \mathbf{I}_{3 \times 3}
\end{array}\right) \dot{\mathbf{q}}+ & k\left(\begin{array}{cc}
2 \mathbf{I}_{3 \times 3} & \mathbf{A} \\
\mathbf{A}^{T} & \mathbf{S}_{22}
\end{array}\right) \mathbf{q} \\
& =\mathbf{Q}^{\prime}\left(\frac{\kappa_{12}}{\kappa_{11}} V_{p z t}(t)\right),
\end{aligned}
$$

where $\mathbf{q}$ is the vector of displacements of the large masses, small masses, and the cantilever tip: $\left[X_{1}, X_{2}, X_{3}, x_{2}, x_{3}, x_{L}\right]^{T} ; \mathbf{M}=\operatorname{diag}\left(M, M, M, m, m, m_{L}\right)$; A is a lower diagonal Toeplitz matrix of -1's; $\mathbf{S}_{\mathbf{2 2}}$ is a matrix of stiffness ratios involving $K / k$ and $k_{L} / k$; and $\mathbf{Q}^{\prime}$ is the vector determining the force input distribution. Because only one unit was ON at a time, the input $V_{p z t}(t)$ is a scalar. The matrix block $\mathbf{S}_{\mathbf{2}}$ and the vector $\mathbf{Q}^{\prime}$ are the only quantities that change with the ON-OFF configuration. For frequency response prediction, the model structure in (29) is readily converted to a transfer function from $V_{p z t}$ to $x_{L}$ using the state $[\mathbf{q} \dot{\mathbf{q}}]^{T}$ and the output equation $x_{L}=\left[\mathbf{0}_{\mathbf{1 \times 5}}, 1, \mathbf{0}_{\mathbf{1} \times \mathbf{6}}\right] \mathbf{q}$.

The parameters of the experimental system used in the theoretical model are given in Table II. The stiffness parameters were measured directly using a Transducer Techniques load cell and the laser displacement sensor described previously. The damping $b_{L}$ and mass $m_{L}$ were identified from an initial condition response of the cantilever, and the ratio $\frac{\kappa_{12}}{\kappa_{11}}$ was obtained from the DC response of the assembled system. As is standard in vibration models the damping is used as a free parameter. Therefore, the damping constant $b$ was tuned to match the experimental data as closely as possible.

\begin{tabular}{|c|c|c|}
\hline \hline Parameter & Value & Units \\
\hline$k$ & 4.1 & $\frac{N}{m m}$ \\
\hline$K$ & 42 & $\frac{N}{m m}$ \\
\hline$k_{L}$ & 2.9 & $\frac{N}{m m}$ \\
\hline$b$ & 0.58 & $\frac{N \cdot s}{m m}$ \\
\hline$b_{L}$ & 2.8 & $\frac{N \cdot s}{m m}$ \\
\hline$m$ & 5.9 & $g$ \\
\hline$M$ & 35 & $g$ \\
\hline$m_{L}$ & 1.8 & $g$ \\
\hline$\frac{\kappa_{12}}{\kappa_{11}}$ & $8.5 \times 10^{-3}$ & $\frac{C}{m}$ \\
\hline \hline \multicolumn{3}{|c}{ TABLE II }
\end{tabular}

THEORETICAL MODEL PARAMETERS

The theoretical model and experimental model are compared in Fig. 10 for both the ON-OFF-OFF case and the OFFON-OFF case. The experimentally obtained gain values and dominant resonant peak locations are well predicted by the linear theoretical model. For the ON-OFF-OFF case, the first 
resonant peak predicted by the model occurred at $f_{n 1}=33$ $\mathrm{Hz}$, while the measured peak occurs at approximately $32 \mathrm{~Hz}$, resulting in a difference of $3 \%$. For the OFF-ON-OFF case, notice that the resonant peak shifted upwards by approximately $8 \mathrm{~Hz}$ since both the theoretical and the experimental results show a fundamental frequency of approximately $41 \mathrm{~Hz}$. In the magnitude curve for the ON-OFF-OFF case, the minor resonant peaks at $26 \mathrm{~Hz}$ and $38 \mathrm{~Hz}$ are likely unmodeled vibration modes in the cantilever. These resonant peaks are well damped and not viewed as a significant source of error in the model order.

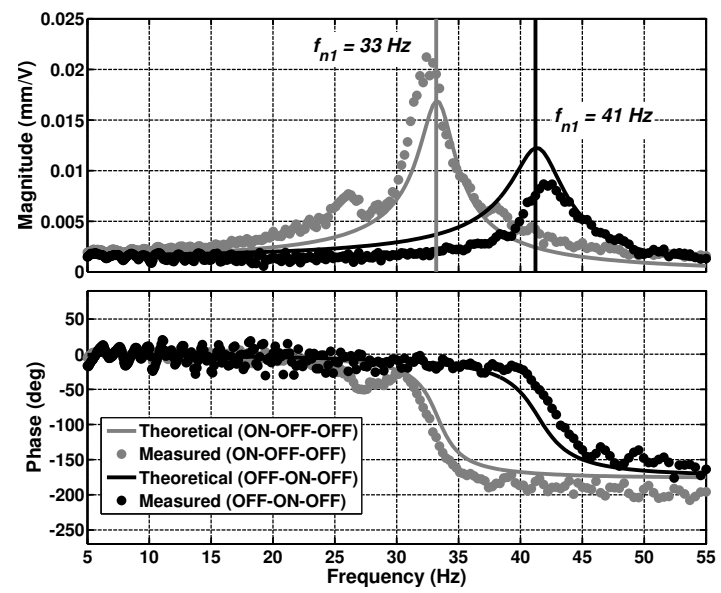

Fig. 10. Comparison of theoretical model and experimental measurement for the ON-OFF-OFF and OFF-ON-OFF configuration.

One important feature of these results is the displacement amplification effect at resonance. The amplification of displacement is an important feature particularly for piezoelectric actuators because their inherent strain is extremely small ( 0.1 $\%$ ). The amplification flexures (Fig. 1) amplify the piezoelectric stack strain to approximately $10 \%$ and tuning the resonant frequency to a desired value allows the actuator to obtain an additional 5 to 10 times larger output displacement. For the first experimental case, the measured ratio of the resonance magnitude to the DC magnitude was 11.1. For the second case, this ratio was 5.7 .

The most important feature of these results is the change in fundamental frequency. Specifically, the increase in fundamental frequency from the first case (ON-OFF-OFF) to the second case (OFF-ON-OFF) illustrates that the resonance properties of the system are indeed tunable and that the tuning may be achieved by simply changing the configuration of the ON-OFF units within the strand.

\section{CONCLUSION}

The two primary objectives of this work were to quantify the basic properties of VSCAs and describe a novel PZT-based design that achieves variable resonant frequencies through variable stiffness. The change in stiffness and resonant frequencies is achieved by selectively turning variable stiffness units ON or OFF within a serial strand. The strands may also exist within a greater parallel structure, which is one subject of future research. Experiments and models both illustrate the shift in resonant frequency based on a simple change in $\mathrm{ON}$ OFF configuration.

The PZT-based actuators considered in this paper show particular promise for VSCAs because they achieve static strain that is commensurate with skeletal muscle (10-20\%) and their strain is further amplified under resonance conditions. Furthermore, VSCA designs based on PZT actuator cells are durable, low power, high bandwidth devices. One particular advantage of PZT is its near-zero power consumption when holding a load in DC. We are presently exploring application areas for our VSCA technology include deep-sea robotics, aircraft manufacturing, and nuclear reactor inspection robots. Further extensions of the technology include using cells with differing stiffnesses to achieve more uniform modal density across the tuning range and using the cellular system as both an actuator and an energy harvesting device for battery recharge in mobile robots.

\section{REFERENCES}

[1] J. Choi, S. Park, W. Lee, and S. Kang, "Design of a Robot Joint with Variable Stiffness," Proc. of IEEE Int. Conf. on Robotics and Automation, pp. 1760-1765, 2008.

[2] J. Hurst, J. Chestnutt, and A. Rizzi, "An Actuator with Physically Variable Stiffness for Highly Dynamic Legged Locomotion," Proc. of IEEE Int. Conf. on Robotics and Automation, pp. 4662-4667, 2004.

[3] R. Blickhan, "The Spring-Mass Model for Running and Hopping," Journal of Biomechanics, Vol. 22, No. 11/12, pp. 1217-1227, 1989.

[4] R. Schiavi, G. Grioli, S. Sen, and A. Bicchi, "VSA-II: A Novel Prototype of Variable Stiffness Actuator for Safe and Performing Robots Interacting with Humans," Proc. of IEEE Int. Conf. on Robotics and Automation, pp. 1760-1765, 2008.

[5] M. Raibert, "Legged Robots That Balance," Cambridge, MA: MIT Press, 1986.

[6] G. Pratt and M. Williamson, "Series Elastic Actuators," Proc. of IEEE/RSJ Int. Conf. on Intelligent Robots and Systems, Vol. 1, pp. 399-406, 1995.

[7] J. Yan, R. Wood, S. Avandhanula, M. Sitti, and R. Fearing, "Towards Flapping Wing Control for a Micromechanical Flying Insect," Proc. of IEEE Int. Conf. on Robotics and Automation, pp. 3901-3908, 2001.

[8] K. Issac and S. Agrawal, "An Investigation Into the Use of Springs and Wing Motions to Minimize the Power Expended by a Pigeon- Sized Mechanical Bird for Steady Flight," Journal of Mech. Design, Vol. 129, pp. 381-389, 2007.

[9] N. Lobontiu, M. Goldfarb, and E. Garcia, "Maximizing the Resonant Displacement of Piezoelectric Beams," Proc. of SPIE, Vol. 3668, No. 154, 1999.

[10] R. Kornbluh, R. Pelrine, J. Eckerle, and J. Joseph, "Electrostrictive Polymer Artificial Muscle Actuators," Proc. of IEEE Int. Conf. on Robotics and Automation, Vol. 3, pp. 2147-2154, 1998.

[11] L. Goodman, M. Riley, and S. Mitra, and M. Turvey, "Advantages of Rhythmic Movements at Resonance: Minimal Active Degrees of Freedom, Minimal Noise, and Maximal Predictability," Journal of Motor Behavior, Vol. 32, No. 1, pp. 3-8, 2000.

[12] V. Challa, et.al, "A Vibration Energy Harvesting Device with Bidirectional Resonance Frequency Tunability," Smart Materials and Structures, Vol. 17, No. 015035, 2008.

[13] K. Cho, J. Rosmarin, and H. Asada, "Design of Vast DOF Artificial Muscle Actuators with a Cellular Array Structure and its Application to a Five-fingered Robotic Hand," Proc. of IEEE Int. Conf. on Robotics and Automation, pp. 2214-2219, 2006.

[14] J. Ueda, T. Secord, and H. Asada, "Design of PZT Cellular Actuators with Power-law Strain Amplification," Proc. of IEEE/RSJ Int. Conf. on Robots and Systems, pp. 1160-1165, 2007.

[15] N. Hogan, "Adaptive Control of Mechanical Impedance by Coactivation of Antagonist Muscles," IEEE Trans. on Auto Cont., Vol. 29, pp. 681-690, 1984.

[16] G.M.L Gladwell, Inverse Problems in Vibration, Martinus Nijhoff, Dordrecht, 1986. 